\title{
Aplicação da Teoria do Dano Concentrado como ferramenta de diagnóstico de estruturas: estudo de caso de varanda colapsada
}

\author{
R. N. Cunha ${ }^{1 *}$, D. V. C. Teles ${ }^{2}$, D. M. Santos ${ }^{2}$, C. S. Vieira ${ }^{2}$, D. L. N. F. Amorim ${ }^{1,2}$ \\ *Autor para contato: rafael.cunha@ctec.ufal.br ${ }^{2}$ Laboratório de Modelagem Matemática em Engenharia Civil, Programa de Pós-Graduação em Engenharia Civil, Universidade Federal de Sergipe, São Cristóvão, Brasil \\ ${ }^{1}$ Programa de Pós-Graduação em Engenharia Civil, Universidade Federal de Alagoas, Maceió, Brasil
}

\section{Resumo}

Inspeções em estruturas são fundamentais para identificar problemas que possam levar a patologias, a fim garantir a vida útil de projeto. $\mathrm{O}$ uso de simulações numéricas permite realizar a identificação de possíveis problemas na estrutura. Como alternativa, a Teoria do Dano Concentrado (TDC) combina os conceitos da mecânica da fratura e do dano clássico em rótulas inelásticas, cuja variável de dano é definida a partir da fissuração do concreto. Desta forma, o presente trabalho avaliou a aplicação da TDC a uma varanda em uso, situada no Brasil, que veio ao colapso. A partir dos resultados da simulação, foi possível avaliar a causa do incidente, além de obter a condição final da laje após o colapso.

Palavras chave: Teoria do Dano Concentrado; Concreto Armado; Ferramenta de Diagnóstico; Varanda.

Cunha, R. N., Teles, D. V. D., Santos, D. M., Vieira, C. S., Amorim, D. L. N. F. 2021, Aplicação da Teoria do Dano Concentrado como ferramenta de diagnóstico de estruturas: estudo de caso de varanda colapsada. Proceedings of XVI Congreso Latinoamericano de Patología de la Construcción y VIII Congreso de Control de Calidad en la Construcción. v. X, pp. X-X. 


\begin{abstract}
Structural inspections are essential to identify problems that can lead to pathologies, in order to guarantee the useful life of the project. The use of numerical simulations allows the identification of possible problems in the structure. As an alternative, the Lumped Damage Mechanics (LDM) combines the concepts of fracture mechanics and classic damage in inelastic hinges, whose damage variable is defined as the cracking of the concrete. Thus, the present paper evaluated the application of LDM to a balcony in use, located in Brazil, which came to collapse. From the results of the simulation, it was possible to assess the cause of the incident, in addition to obtaining the final condition of the slab after the collapse.
\end{abstract}

Keywords: Lumped Damage Mechanics; Reinforced Concrete; Diagnosis tool; Balcony.

\title{
Resumen
}

Las inspecciones estructurales son fundamentales para identificar problemas que pueden derivar en patologías, con el fin de garantizar la vida útil del proyecto. El uso de simulaciones numéricas permite la identificación de posibles problemas en la estructura. Como alternativa, la Mecánica de Daño Concentrado (MDC) combina los conceptos de mecánica de fractura y daño clásico en rotulas inelásticas, cuya variable de daño se define como el agrietamiento del concreto. Así, el presente trabajo evaluó la aplicación de MDC a un balcón en uso, ubicado en Brasil, que llegó al colapso. A partir de los resultados de la simulación se pudo valorar la causa del incidente, además de obtener el estado final de la losa tras el colapso.

Palabras clave: Mecánica de daño concentrado; Concreto reforzado; Herramienta de diagnóstico; Balcón.

\section{INTRODUÇÃO}

Todas as estruturas possuem uma vida útil estabelecida na etapa de projetos. Com o intuito de garantir esse período, devem ser realizadas obras de manutenção e inspeção a intervalos de tempo regulares. Estes tipos de observações permitem avaliar e corrigir patologias antes que as mesmas se agravem. Para estruturas de concreto armado, as inspeções periódicas objetivam identificar e aferir desde a degradação do concreto até a condição das armaduras. Caso as medidas corretivas necessárias não sejam investigadas e executadas, a estrutura pode vir a um colapso parcial ou total. O estudo da condição das estruturas pode ser realizado em campo, através de métodos numéricos ou com a combinação de métodos experimentais e numéricos. Para o primeiro caso, é possível utilizar de métodos não destrutivos como radares de penetração no solo (Solla et al., 2012) ou emissão acústica (Noorsuhada, 2016), ou métodos destrutivos com o uso de explosões (Ruggiero et al., 2019). A combinação de métodos experimentais e numéricos permite verificar a condição da estrutura através de ensaios, com posterior reprodução da mesma com o uso de métodos numéricos. Este tipo de análise permite obter mais parâmetros e condições da estrutura a partir das simulações desenvolvidas, como a obtenção dos níveis de dano e as propriedades dinâmicas (Masciotta et al., 2019). No entanto, a aplicação de métodos experimentais pode se tornar inviável, devido a condições como o acesso a estrutura, tempo ou custos envolvidos. Desta forma o uso de métodos numéricos supre estes problemas, sendo o método dos elementos finitos uma das alternativas mais 
utilizadas para a análise de estruturas (Tang et al., 2005; Wu et al., 2020; Abedeni et al., 2021; Tenorio-Monteiro e Juárez-Luna, 2021). No entanto a aplicação deste método pode apresentar desvantagens, como o alto custo computacional requerido para realizar as simulações.

Como uma alternativa viável para a análise de estruturas de concreto armado surgiu a Teoria do Dano Concentrado (TDC). Esta teoria combina os conceitos da mecânica da fratura e do dano clássico em rótulas plásticas, a qual define a fissuração do concreto como a variável de dano. Esta teoria foi inicialmente formulada para estruturas de concreto armado sujeitos a cargas sísmicas (Flórez-López, 1993; Cipollina et al., 1995; Perdomo et al., 1999; Rajasankar et al., 2009; Perdomo et al., 2013; Teles et al., 2021) e expandida para outras condições, como cargas de impacto (Yang e Wang, 2010; Teles et al. 2020; Oliveira et al., 2020) e revestimentos de túneis (Amorim et al., 2013; Amorim et al., 2014; Brito et al., 2020).

O uso da TDC tem como vantagens o baixo custo computacional exigido para realizar as análises, assim como a fácil determinação dos parâmetros do modelo para estruturas de concreto armado, como o momento de fissuração e as condições de carregamento. Flórez-López et al. (2015) define a integridade de estruturas e necessidades de manutenção de acordo com o valor da variável de dano nos elementos estruturais. Para uma estrutura de concreto armado, o valor de dano na faixa de 0,3 a 0,4 representa o início do escoamento da armadura e, para uma condição de dano acima de 0,6 , o elemento atingiu sua capacidade máxima de carga.

Portanto, o objetivo deste trabalho é utilizar a Teoria do Dano Concentrado como uma ferramenta de diagnóstico, com o uso da variável de dano para avaliar a estrutura de uma varanda que veio ao colapso durante seu uso.

\section{TEORIA DO DANO CONCENTRADO}

As variáveis de problemas estruturais podem ser classificadas como cinemáticas, estáticas ou esforços internos, as quais estão relacionadas através das equações cinemáticas, equações de equilíbrio e as leis constitutivas. Considerando uma estrutura de pórtico, no plano cartesiano de referência XZ, a matriz de deslocamentos generalizados $\{\mathbf{U}\}$ e a matriz de forças externas generalizadas $\{\mathbf{P}\}$ podem ser descritas de acordo com as Equações (1) e (2), respectivamente:

$$
\begin{aligned}
\{\mathbf{U}\} & =\left\{\begin{array}{llllllllllllllll}
u_{1} & w_{1} & \theta_{1} & \ldots & u_{i} & w_{i} & \theta_{i} & \ldots & u_{j} & w_{j} & \theta_{j} & \ldots & u_{n} & w_{n} & \theta_{n}
\end{array}\right\}^{T} \\
\{\mathbf{P}\} & =\left\{\begin{array}{lllllllllllllll}
P_{u 1} & P_{w 1} & P_{\theta 1} & \ldots & P_{u i} & P_{w i} & P_{\theta i} & \ldots & P_{u j} & P_{w j} & P_{\theta j} & \ldots & P_{u n} & P_{w n} & \left.P_{\theta n}\right\}^{T}
\end{array}\right.
\end{aligned}
$$

em que $u_{1}$ é o deslocamento do nó 1 na direção paralela ao eixo $\mathrm{X}, w_{1}$ é o deslocamento do nó 1 na paralela do eixo $\mathrm{Z}, \theta_{l}$ é a rotação do nó 1 no plano $\mathrm{XZ}, P_{u n}$ é a força aplicada no nó n na direção paralela ao eixo $\mathrm{X}, P_{w n}$ é a força aplicada no nó n na direção paralela ao eixo $\mathrm{Z}, P_{\theta n}$ é o momento aplicado no nó n no plano XZ e o subscrito " $T$ ” refere-se a transposta do vetor.

A dedução clássica de elementos finitos relaciona diretamente as matrizes $\{\mathbf{U}\}$ e $\{\mathbf{P}\}$. No entanto a presente abordagem realiza um procedimento alternativo, com o uso de mais matrizes, apresentados a seguir. Para um elemento de pórtico, as equações de equilíbrio podem ser descritas de acordo com a Equação (3): 
$\{\mathbf{P}\}=\sum_{b=1}^{m}[\mathbf{B}]_{b}^{T}\{\mathbf{M}\}_{b}$

sendo $[\mathbf{B}]_{b}$ a matriz de transformação e $\{\mathbf{M}\}_{b}$ a matriz de esforços generalizados do elemento finito, dados pela Equação (4) e (5), respectivamente.

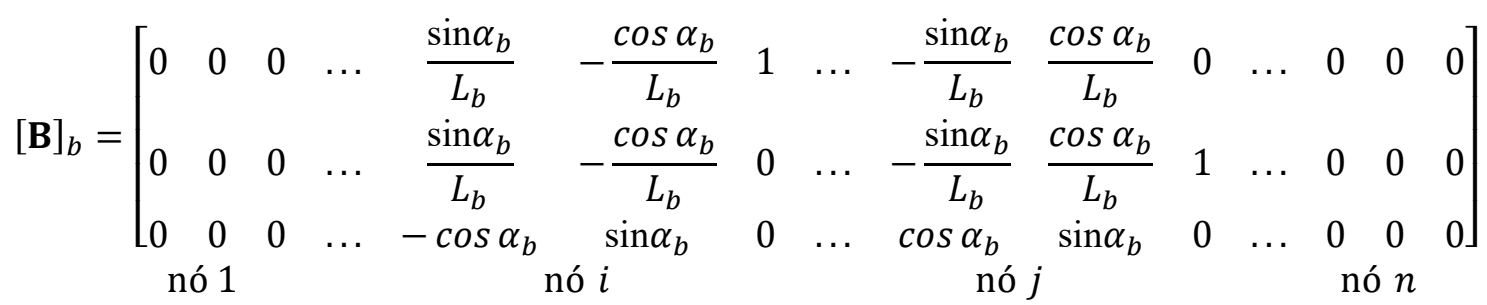

$$
\{\mathbf{M}\}_{b}=\left\{\begin{array}{lll}
M_{i} & M_{j} & N
\end{array}\right\}^{T}
$$

em que $M_{i}$ e $M_{j}$ são os momentos fletores nos nós $i$ e $j$, respectivamente, $N$ é o esforço normal no elemento, $L_{b}$ e $\alpha_{b}$ são o comprimento e a orientação do elemento, respectivamente (Figura 1).
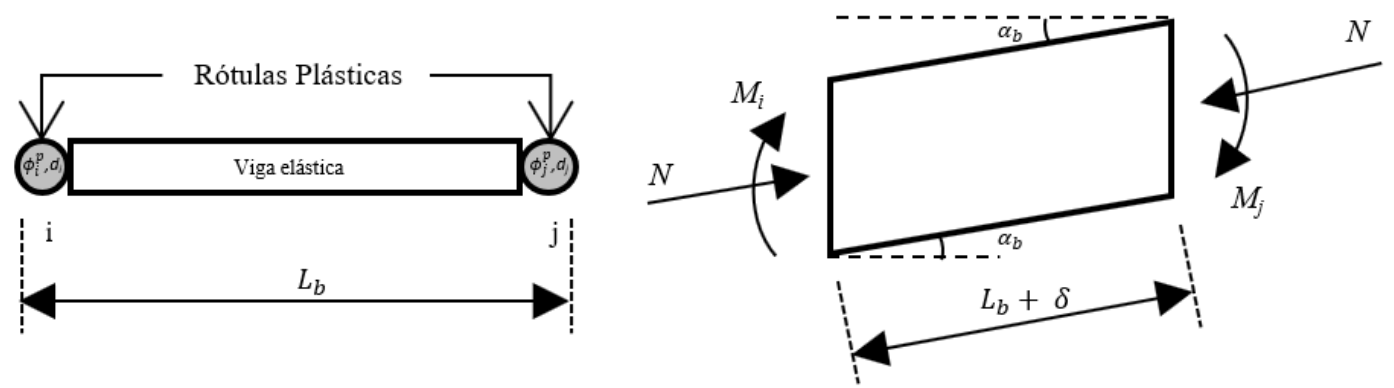

Figura 1. Definição geométrica e esforços generalizados para um elemento de pórtico. Fonte: Perdomo et al. (2013).

As equações cinemáticas podem ser definidas de acordo com o Equação (6):

$$
\{\dot{\boldsymbol{\Phi}}\}_{b}=[\mathbf{B}(\mathbf{U})]_{b}\{\dot{\mathbf{U}}\}
$$

onde $[\mathbf{B}(\mathbf{U})]_{b}$ é a mesma matriz de transformação apresentada na Equação (4), porém admitindo grandes deslocamentos e $\{\boldsymbol{\Phi}\}$ é a matriz de deslocamentos generalizados, conjugados à matriz $\{\mathbf{M}\}$, e definida através da Equação (7):

$$
\{\boldsymbol{\Phi}\}_{b}=\left\{\begin{array}{lll}
\phi_{i} & \phi_{j} & \delta
\end{array}\right\}
$$

sendo $\phi_{i}$ e $\phi_{j}$ as rotações devido aos momentos fletores $M_{i}$ e $M_{j}$, respectivamente, e $\delta$ é a deformação axial devido ao esforço normal $N$. 
Se a hipótese de pequenos deslocamentos e deformações for assumida, a Equação (7) pode ser reescrita como a Equação (8), em que $\left[\mathbf{B}_{0}\right]_{b}$ é a matriz de transformação para a condição inicial do elemento.

$$
\{\boldsymbol{\Phi}\}_{b}=\left[\mathbf{B}_{0}\right]_{b}\{\mathbf{U}\}
$$

As relações elásticas do elemento são obtidas através da hipótese de equivalência de deformações, aplicando o Teorema de Castigliano (Amorim et al., 2013; Amorim et al., 2014; Flórez-López et al., 2015). Além disso, é admitido que os fenômenos inelásticos do elemento são concentrados nas rótulas, localizadas nas extremidades do mesmo (Figura 1).

$$
\left\{\boldsymbol{\Phi}-\boldsymbol{\Phi}^{p}\right\}_{b}=[\mathbf{F}(\mathbf{D})]_{b}\{\mathbf{M}\}_{b}
$$

em que $\left\{\boldsymbol{\Phi}^{p}\right\}_{b}$ é a matriz de deformações plásticas generalizadas e $[\mathbf{F}(\mathbf{D})]_{b}$ é a matriz de flexibilidade com dano do elemento, dadas através da Equação (10) e (11), respectivamente:

$$
\begin{aligned}
\left\{\boldsymbol{\Phi}^{p}\right\}_{b} & =\left\{\begin{array}{lll}
\phi_{i}^{p} & \phi_{j}^{p} & 0
\end{array}\right\}^{T} \\
{[\mathbf{F}(\mathbf{D})]_{b} } & =\left[\begin{array}{ccc}
\frac{L_{b}}{3 E I\left(1-d_{i}\right)} & -\frac{L_{b}}{6 E I} & 0 \\
-\frac{L_{b}}{6 E I} & \frac{L_{b}}{3 E I\left(1-d_{j}\right)} & 0 \\
0 & 0 & \frac{L_{b}}{A E}
\end{array}\right]
\end{aligned}
$$

sendo $d_{i}$ e $d_{j}$ as variáveis de dano nas rótulas $i$ e $j$, respectivamente e $E I$ e $A E$ são as rigidezes a flexão e axial do elemento, respectivamente.

Com o intuito de complementar a lei constitutiva, as variáveis internas do problema (dano e rotação plástica) precisam ser consideradas. Portanto, a energia complementar do elemento finito é admitida de acordo com a equação (12).

$$
W=\frac{1}{2}\{\mathbf{M}\}_{b}^{T}\left\{\boldsymbol{\Phi}-\boldsymbol{\Phi}^{\mathrm{p}}\right\}_{b}
$$

As leis de evolução das variáveis de dano são obtidas através do critério de Griffith:

$$
\begin{aligned}
& \left\{\begin{array}{lll}
\Delta d_{i}>0 & \Rightarrow & G_{i}=Y\left(d_{i}\right) \\
G_{i}<Y\left(d_{i}\right) & \Rightarrow & \Delta d_{i}=0
\end{array} \quad \therefore G_{i}=\frac{\partial W}{\partial d_{i}}=\frac{M_{i}^{2} F_{11}^{0}}{2\left(1-d_{i}\right)^{2}}\right. \\
& \left\{\begin{array}{lll}
\Delta d_{j}>0 & \Rightarrow & G_{j}=Y\left(d_{j}\right) \\
G_{j}<Y\left(d_{j}\right) & \Rightarrow & \Delta d_{j}=0
\end{array} \quad \therefore G_{j}=\frac{\partial W}{\partial d_{j}}=\frac{M_{j}^{2} F_{22}^{0}}{2\left(1-d_{j}\right)^{2}}\right.
\end{aligned}
$$


em que $G_{i}$ e $G_{j}$ são os momentos de condução da fissura para as rótulas $i$ e $j$, respectivamente, e $Y\left(d_{i}\right)$ e $Y\left(d_{j}\right)$ são as funções de resistência a fissuração.

Para elementos de concreto armado, a função de resistência a fissuração é dada através da Equação (14):

$$
Y\left(d_{i}\right)=Y_{0}+q \frac{\ln \left(1-d_{i}\right)}{1-d_{i}}
$$

sendo $Y_{0}$ e $q$ parâmetros do modelo.

As leis de evolução plástica para cada rótula do elementos são dadas pela Equação (15):

$$
\begin{aligned}
& \left\{\begin{array}{llll}
\Delta \phi_{i}^{p}>0 & \Rightarrow & f_{i}=0 & \therefore f_{i}=\left|\frac{M_{i}}{1-d_{i}}-C \phi_{i}^{p}\right|-k_{0} \leq 0 \\
f_{i}<0 & \Rightarrow & \Delta \phi_{i}^{p}=0 &
\end{array}\right. \\
& \left\{\begin{array}{lll}
\Delta \phi_{j}^{p}>0 & \Rightarrow f_{j}=0 & \therefore f_{j}=\left|\frac{M_{j}}{1-d_{j}}-C \phi_{j}^{p}\right|-k_{0} \leq 0 \\
f_{j}<0 & \Rightarrow & \Delta \phi_{j}^{p}=0
\end{array}\right.
\end{aligned}
$$

onde $f_{i}$ e $f_{j}$ as funções de escoamento para as rótulas i e j, respectivamente, e $C$ e $k_{0}$ são parâmetros do modelo. Os parâmetros $Y_{0}, q, C$ e $k_{0}$ estão diretamente relacionados a teoria clássica do concreto armado como mostrado por Permodo et al. (1999), Amorim et al. (2013), Amorim et al. (2014), Flórez-López et al. (2015) e Brito et al. (2020).

\section{ESTUDO DE CASO: COLAPSO DE UMA VARANDA}

Foi analisada uma varanda de um edifício situado na cidade do Rio de Janeiro que, 15 anos após sua construção, veio ao colapso. A laje da varanda sofreu uma fissuração severa na região de seu apoio, o que acarretou uma rotação de aproximadamente $17^{\circ}$, mas que permaneceu conectada a estrutura do edifício, como mostrado na Figura 2.

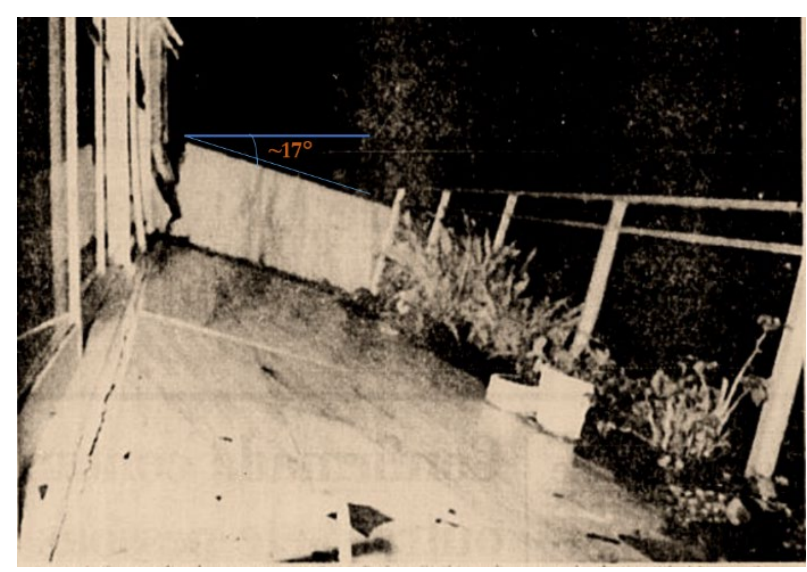

Figura 2. Laje da varanda após colapso. Fonte: Jornal do Brasil (1993). 
A estrutura da varanda era uma laje de concreto armado maciça, com $12 \mathrm{~cm}$ de espessura, largura de $203 \mathrm{~cm}$ e comprimento de $932 \mathrm{~cm}$. Esta laje estava engastada na laje da sala de estar, cuja espessura era igual a $15 \mathrm{~cm}$ (Figura 3). A face inferior de ambas as lajes estavam no mesmo nível, como mostrado na Figura 4, desta forma resultando em uma diferença de nível de $3 \mathrm{~cm}$ na face superior (Lima, 1996).

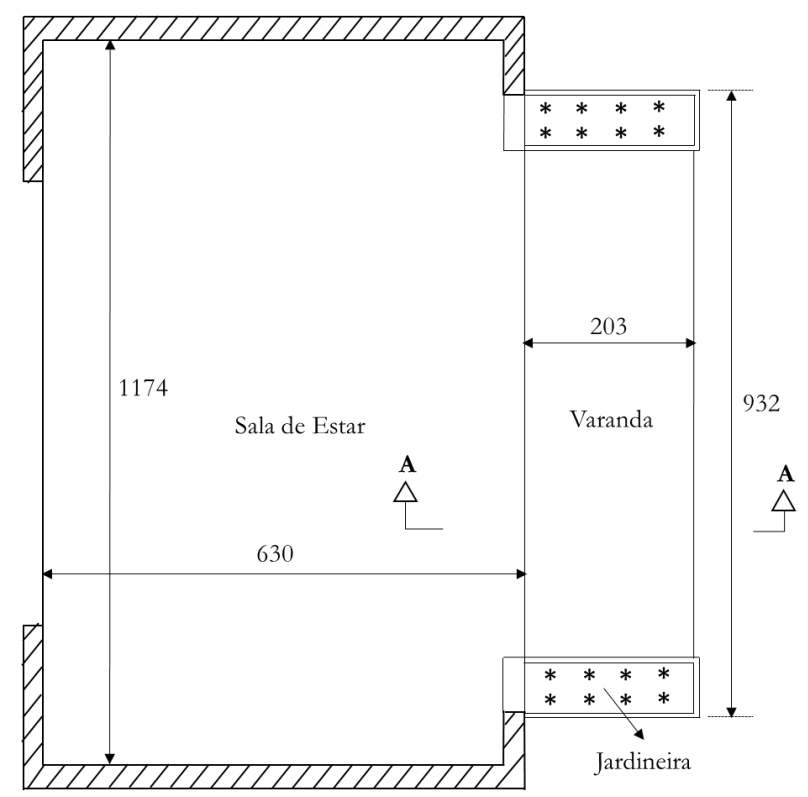

Figura 3. Dimensões da sala de estar e varanda, em centímetros. Fonte: Lima (1996).

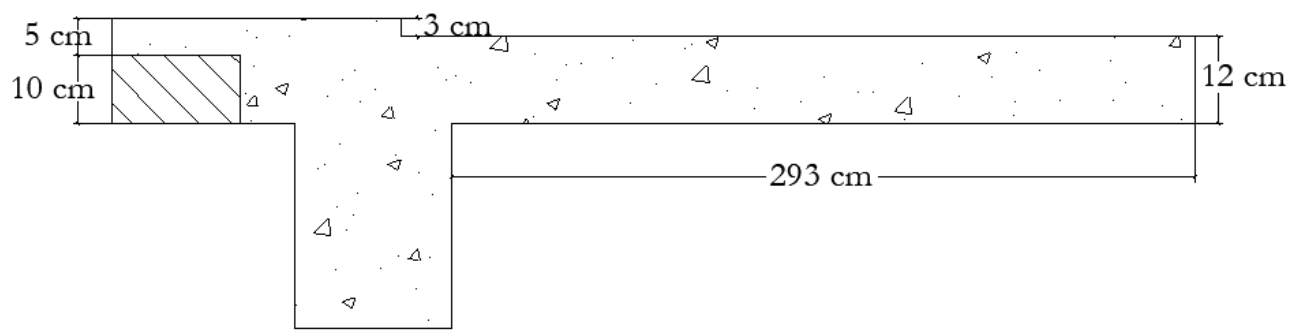

Figura 4. Seção transversal da laje. Fonte: Lima (1996).

Após inspeções realizadas no local, foi observado que as barras de aço permaneceram intactas, não apresentavam sinais de corrosão, possuíam diâmetro de $9,5 \mathrm{~mm}$ e estavam espaçadas de $15 \mathrm{~cm}$. Foram extraídos de campo 7 corpos de prova de concreto com o uso de uma sonda rotativa e 3 amostras das barras de aço utilizadas na armadura negativa para posterior análise. Através de ensaios de compressão simples e de tração foi determinada a resistência à compressão do concreto $\left(f_{c k}\right)$ igual a $30 \mathrm{MPa}(\mathrm{C} 30)$ e a resistência à tração do aço igual a $500 \mathrm{MPa}(\mathrm{CA}-50)$. Durante o incidente do colapso, foi observado que não havia nenhum tipo de sobrecarga na laje da varanda. As cargas permanentes eram as únicas atuantes na estrutura, referentes ao peso próprio do concreto $\left(3,0 \mathrm{kN} / \mathrm{m}^{2}\right)$, ao revestimento inferior $\left(0,4 \mathrm{kN} / \mathrm{m}^{2}\right)$, à argamassa de assentamento $\left(1,0 \mathrm{kN} / \mathrm{m}^{2}\right)$ e ao piso de mármore $\left(0,56 \mathrm{kN} / \mathrm{m}^{2}\right)$ (Lima, 1996). As ações permanentes combinadas totalizam um carregamento igual a $4,96 \mathrm{kN} / \mathrm{m}^{2}$, o qual provocou um momento fletor negativo na seção do engaste igual a $10,22 \mathrm{kNm}$. 
A laje foi dimensionada para resistir apenas às ações permanentes de projeto, considerando para fins de cálculo um concreto com $f_{c k}$ igual a $14 \mathrm{MPa}$. Desta forma, a seção do engaste da laje foi dimensionada com uma área de aço total de $3,4 \mathrm{~cm}^{2} / \mathrm{m}$. No entanto, a partir das observações realizadas em campo, foi observado que a área total de aço utilizada foi igual a $4,72 \mathrm{~cm}^{2} / \mathrm{m}$ (Lima, 1996).

Portanto, com base nas observações realizadas em campo, foi concluído que o colapso da varanda ocorreu devido a repentina retificação das barras de aço da armadura negativa no engaste, a qual foi executada com dupla curvatura (Figura 5). Este tipo de acidente ocorre devido a tendência das barras retornarem a seu estado inicial, a qual não é resistida pela camada de cobrimento do concreto. Desta forma, o concreto na região da ligação é expulso no momento que ocorre a retificação das barras de aço, causando o fenômeno denominado empuxo no vazio.

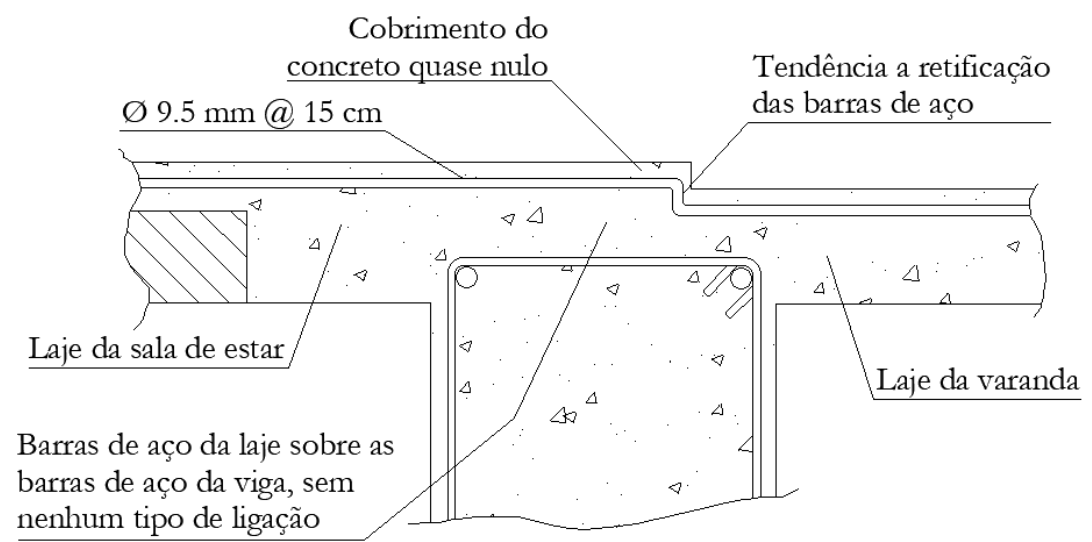

Figura 5. Detalhamento da seção da laje antes do acidente. Fonte: Lima (1996).

\section{RESULTADOS}

A verificação da condição dos esforços na laje pode ser realizada através dos procedimentos presentes na NBR 6118 (2014), com o cálculo do momento de fissuração do concreto, determinado através da Equação (16).

$$
M_{c r}=\frac{0,315 f_{c k}^{\frac{2}{3}} b_{w} h^{3}}{12 y_{t}}
$$

em que $y_{\mathrm{t}}$ é a distância do centro de gravidade da seção à fibra mais tracionada e $b_{w}$ e $h$ são a base e a altura da seção transversal, respectivamente.

Desta forma, é possível verificar que o momento de fissuração da laje é igual a 15,72 kNm, valor superior ao momento fletor atuante no apoio da laje $(10,22 \mathrm{kNm})$. Esta verificação também pode ser realizada com o uso da TDC, na qual deve ser observada a lei de evolução do dano (Equação (13)) da rótula desejada. Ao realizar a simulação, é possível verificar que $G<Y(d)$ e, portanto, o nível de carregamento atuante não causaria dano na seção do engaste. Desta forma, é possível corroborar que o acidente ocorreu devido a retificação das barras de aço (empuxo no vazio). 
As análises de campo permitiram observar a condição final da laje após o acidente, verificando que a rotação final da estrutura foi de aproximadamente $17^{\circ}$ e que a espessura residual de concreto que conectava a laje da varanda a laje da sala de estar era de aproximadamente $2 \mathrm{~cm}$, como mostrado na Figura 6.

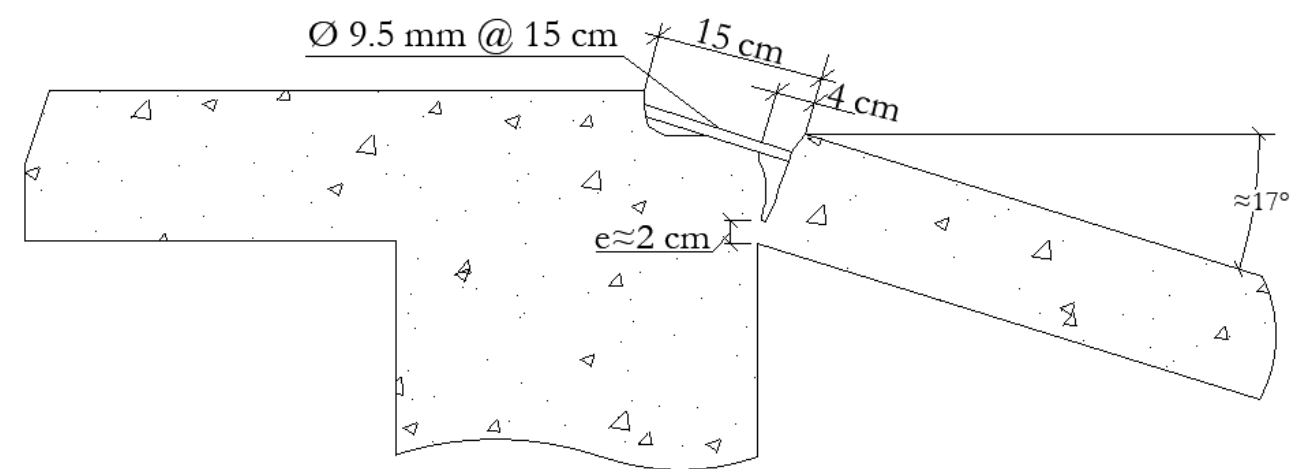

Figura 6. Detalhamento da seção da laje depois do acidente. Fonte: Lima (1996).

A simulação da laje da varanda, utilizando a TDC, foi realizada admitindo a hipótese de grandes deslocamentos, obtendo um valor de dano igual 0,9995, caracterizando um comportamento estrutural inaceitável (Florez-Lopez et al., 2015). Nesta condição, os deslocamentos vertical e horizontal na borda da laje foram iguais a $58,99 \mathrm{~cm}$ e $13,57 \mathrm{~cm}$, respectivamente, os quais levam a uma rotação de aproximadamente $17,30^{\circ}$. É possível observar que os resultados obtidos numericamente apresentam valores próximos aos observados in loco, como o ângulo final da laje $\left(\approx 17^{\circ}\right)$. Além disso, como observado por Amorim et al. (2014), o valor de dano penaliza o momento de inércia diretamente, desta forma, a espessura restante na seção do engaste pode ser estimada em aproximadamente $1 \mathrm{~cm}$, valor próximo ao observado em campo $(\approx 2 \mathrm{~cm})$.

\section{CONCLUSÕES}

A análise de estruturas total ou parcialmente colapsadas pode ser desenvolvida com o uso da Teoria do Dano Concentrado (TDC). O presente trabalho realizou o estudo de uma laje de concreto armado de uma varanda, que veio ao colapso após 15 anos de serviço.

A aplicação da TDC permitiu observar que o incidente ocorrido com a estrutura da varanda não adveio devido a problemas da resistência dos materiais $(G<Y(d))$, sendo o principal motivo a má escolha de detalhamento para a ancoragem da laje da varanda na laje da sala de estar (Figura 5). Este tipo de detalhamento acarretou na tendência a retificação das barras de aço, situação em que o cobrimento de concreto não resistiu aos esforços gerados, denominado empuxo no vazio. Através da simulação desenvolvida utilizando a TDC foi obtido o valor de dano na ligação entre as lajes igual a 0,99995 , caracterizando o colapso. Além disso, o ângulo final de rotação da estrutura foi determinado igual a $17,3^{\circ}$, próximo ao observado em campo $\left(\approx 17^{\circ}\right)$ e a espessura residual de concreto igual a aproximadamente $1 \mathrm{~cm}$, sendo observado em campo a espessura de $2 \mathrm{~cm}$.

Portanto, é possível notar a viabilidade do uso da TDC como ferramenta de análise para estruturas, a fim de determinar seu nível de danificação, a necessidade de medidas corretivas e a segurança para o seu uso.

\section{AGRADECIMENTOS}


O primeiro e o quinto autores agradecem ao Programa de Pós-Graduação em Engenharia Civil da Universidade Federal de Alagoas (PPGEC/UFAL) pelo aporte. O primeiro autor agradece a Coordenação de Aperfeiçoamento de Pessoal de Nível Superior (CAPES) pelo suporte financeiro durante seu curso de doutorado. O segundo autor agradece a CAPES pelo suporte financeiro durante seu curso de mestrado. A quarta autora agradece a PNPD/CAPES pelo suporte financeiro durante seu curso de pós-doutorado.

\section{REFERENCIAS}

Abedeni, M. and Zhang, C.W. (2021), Dynamic vulnerability assessment and damage prediction of RC columns subjected to severe impulsive loading. Structural Engineering and Mechanics. 77(4), pp. 441-461. http://doi.org/10.12989/sem.2021.77.4.441.

Amorim, D.L.N.F., Proença, S.P.B. and Flórez-López, J. (2013), A model of fracture in reinforced concrete arches based on lumped damage mechanics. International Journal of Solids and Structures. 50(24), pp. 4070-9. http://doi.org/10.1016/j.ijsolstr.2013.08.012.

Amorim, D.L.N.F., Proença, S.P.B. and Flórez-López, J. (2014), Simplified modeling of cracking in concrete: Application in tunnel linings. Engineering Structures. 70, pp. 23-35. http://doi.org/10.1016/j.engstruct.2014.03.031.

Associação Brasileira de Normas Técnicas (ABNT). (2014). NBR 6118: Projeto de estruturas de concreto - Procedimento. Rio de Janeiro.

Brito, T.I.J., Santos, D.M., Santos, F.A.S., Cunha, R.N. and Amorim, D.L.N.F. (2020), On the lumped damage modelling of reinforced concrete beams and arches. Frattura ed Integrità Strutturale. 54, pp. 1-20. http://doi.org/10.3221/IGF-ESIS.54.01.

Cipollina, A., López-Inojosa, A. and Flórez-López, J. (1995), A simplified damage mechanics approach to nonlinear analysis of frames. Computers and Structures. 54(6), pp. 1113-26. http://doi.org/10.1016/00457949(94)00394-I.

Flórez-López J. (1993), Modelos de daño concentrado para la simulación del colapso de pórticos planos. Revista Internacional de Metodos Numericos para Calculo y Diseno en Ingenieria. 9(2), pp. 123-139.

Flórez-López, J., Marante, M.E., Picón, R. (2015). Fracture and damage mechanics for structural engineering of frames: State-of-the-art industrial applications, Engineering Science Reference. ISBN: 9781466663794.

Jornal do Brasil, Rio de Janeiro, 21/Março/1993, p. 23.

Lima, N.A. (1996). Empuxo no vazio provoca o colapso da estrutura de uma varanda, In: "Acidentes Estruturais na Construção Civil’, PINI, São Paulo, p. 212. ISBN: 978-8572660617.

Masciotta, M.G., Pellegrini, D., Brigante, D., Barontini, A., Lourenço, P.B., Girardi, M., Padovani, C. and Fabbrocino, G. (2019), Dynamic characterization of progressively damaged segmental masonry arches with one settled support: experimental and numerical analyses. Frattura ed Integrità Strutturale. 14(51), pp. 42341. http://doi.org/10.3221/IGF-ESIS.51.31.

Noorsuhada, M.N. (2016), An overview on fatigue damage assessment of reinforced concrete structures with the aid of acoustic emission technique. Construction and Building Materials. 112, pp. 424-439. http://doi.org/10.1016/j.conbuildmat.2016.02.206.

Oliveira, M.C., Teles, D.V.C. and Amorim, D.L.N.F. (2020), Shear behaviour of reinforced concrete beams under impact loads by the Lumped Damage framework. Frattura ed Integrità Strutturale. 53, pp. 13-25. http://doi.org/10.3221/IGF-ESIS.53.02.

Perdomo ME, Picón R, Marante ME, Hild F, Roux S, Florez-Lopez J (2013), Experimental analysis and mathematical modeling of fracture in RC elements with any aspect ratio. Engineering Structures. 46:407416. http://doi.org/10.1016/j.engstruct.2012.07.005.

Perdomo, M.E., Ramírez, A. and Flórez-López, J. (1999), Simulation of damage in RC frames with variable axial forces. Earthquake Engineering Structural Dynamics. 28(3), pp. 311-28. http://doi.org/10.1002/(sici)1096-9845(199903)28:3<311::aid-eqe819>3.0.co;2-d. 
Rajasankar, J., Iyer, N.R. and Prasad, A.P. (2009), Modelling inelastic hinges using CDM for nonlinear analysis of reinforced concrete frame structures. Computers and Concrete. 6(4), pp. 319-41. http://doi.org/10.12989/cac.2009.6.4.319.

Ruggiero, A., Bonora, N., Curiale, G., De Muro, S., Iannitti, G., Marfia, S., Sacco, E., Scafati, S. and Testa, G. (2019), Full scale experimental tests and numerical model validation of reinforced concrete slab subjected to direct contact explosion. International Journal of Impact Engineering. 132, pp. 103309. http://doi.org/10.1016/j.ijimpeng.2019.05.023.

Solla, M., Lorenzo, H., Novo, A. and Caamaño, J. (2012), Structural analysis of the Roman Bibei bridge (Spain) based on GPR data and numerical modelling. Automation in Construction. 22, pp. 334-339. http://doi.org/10.1016/j.autcon.2011.09.010.

Tang, X.S., Zhang, J.R., Li, C.X., Xu, F.H. and Pan, J. (2005), Damage analysis and numerical simulation for failure process of a reinforced concrete arch structure. Computers and Structures. 83, pp. 2609-2631. http://doi.org/10.1016/j.compstruc.2005.03.017.

Teles, D.V.C., Cunha, R.N., Amorim, D.L.N.F, Picón, R. and López, J.F. (2021), Parametric study of dynamic behaviour of RC dual system design with the Brazilian Standard Code using the lumped damage model. Journal of the Brazilian Society of Mechanical Sciences and Engineering. 43(5), pp. 246. http://doi.org/10.1007/s40430-021-02977-8.

Teles, D.V.C., Oliveira, M.C. and Amorim, D.L.N.F. (2020), A simplified lumped damage model for reinforced concrete beams under impact loads. Engineering Structures. 205. http://doi.org/10.1016/j.engstruct.2019.110070.

Tenorio-Monteiro, E. and Juárez-Luna, G. (2021), Beam-column finite element with embedded discontinuities for modelling damage in reinforced concrete prismatic elements. Structures. 29, pp. 19341953. http://doi.org/10.1016/j.istruc.2020.12.055.

Wu, J., Zhou, Y.M., Zhang, R., Liu, C.B. and Zhang, Z.C. (2020), Numerical simulation of reinforced concrete slab subjected to blast loading and the structural damage assessment. Engineering Failure Analysis. 118, pp. 104926. http://doi.org/10.1016/j.engfailanal.2020.104926.

Yang, T. -S. and Wang, J. -L. (2010), Damage analysis of three-dimensional frame structure suffering from impact. Journal of Vibration and Shock. 29(12), pp. 177-80. 